\title{
Pediatric Pulmonary Artery Rehabilitation: A Review of Our Experience and a Novel Approach Using Bronchial Blockers
}

\author{
Peter D. Winch ${ }^{1}$ - Dmitry Tumin ${ }^{1}$. Jeffrey Moore ${ }^{2} \cdot$ Samantha J. Vizzini $^{3} \cdot$ Darren P. Berman $^{4} \cdot$ Aymen N. Naguib $^{1}$
}

Received: 13 October 2017 / Accepted: 2 May 2018 / Published online: 9 May 2018

(c) Springer Science+Business Media, LLC, part of Springer Nature 2018

\begin{abstract}
Recent advances in the field of pediatric interventional cardiology have resulted in therapies for patients in need of augmented pulmonary artery (PA) flow. Catheter-based PA rehabilitation can be performed safely but not without the potential risk of pulmonary hemorrhage. When severe, this bleeding has the ability to contaminate the bronchi and trachea, and possibly occlude the endotracheal tube. This can result in a critical inability to ventilate and oxygenate these patients. Herein, we review our institutional experience with pulmonary hemorrhage associated with these procedures and the feasibility of predicting this outcome. The secondary aim was to discuss our novel anesthetic approach to a select group of these patients, involving the use of bronchial blockers to preemptively prevent contamination of bilateral bronchi and occlusion of the endotracheal tube.
\end{abstract}

Keywords Airway management $\cdot$ Angiography $\cdot$ Bronchi $\cdot$ Cardiac catheterization $\cdot$ Lung disease

\section{Introduction}

Children with diminutive pulmonary arteries are often given a disheartening prognosis. Through clinical experience, physicians have learned that they represent an especially challenging patient population. Patients with Tetralogy of Fallot (TOF) combined with pulmonary atresia are especially afflicted with this condition, as are patients with underlying Pulmonary Artery (PA) Stenosis, Transposition of the Great Arteries (TGA), Truncus Arteriosus, and Double Outlet Right Ventricle (DORV). Advances in surgical

Electronic supplementary material The online version of this article (https://doi.org/10.1007/s00246-018-1888-6) contains supplementary material, which is available to authorized users.

Peter D. Winch

peter.winch@nationwidechildrens.org

1 Department of Anesthesiology and Pain Medicine, Nationwide Children's Hospital, 700 Children's Drive, Columbus 43205, USA

2 Department of Cardiology, Toledo Children's Heart Center, Toledo, USA

3 Department of Anesthesiology and Perioperative Medicine, Medical University of South Carolina, Charleston, USA

4 Department of Cardiology, Nationwide Children's Hospital, Columbus, USA techniques and improved surgical outcomes for such patients has resulted in improved survival and an increased population of patients presenting for cardiac catheterization in need of detailed assessment of pulmonary blood flow and in many cases a need for increased PA flow [1,2]. Furthermore, in addition to having abnormal pulmonary vasculature at birth, many of these children have abnormal vascular growth, which necessitates early and frequent interventions.

Recent advances in the field of pediatric interventional cardiology have resulted in an enhanced ability to offer therapies to patients in need of augmented PA flow. Catheter-based options include balloon dilation of the pulmonary vasculature with both conventional and cutting balloons and the placement of intravascular stents to maintain arterial patency. These stents are then often further dilated during repeat catheterization. These procedures are collectively described as "PA rehabilitation" and have a defined goal of: (1) improving pulmonary blood flow with growth of the pulmonary arterial vasculature and (2) decreasing the right ventricular pressure load. In the subset of uncorrected or palliated children with existing intra-cardiac shunts, this increase in pulmonary flow often increases systemic saturation by decreasing the degree of right to left shunting.

Catheter-based PA rehabilitation can be performed safely but not without the potential risk of pulmonary hemorrhage. When severe, this bleeding has the ability to contaminate 
the bronchi and trachea, and possibly occlude the endotracheal tube. This can result in a critical inability to ventilate and oxygenate these patients. This may be caused from mechanical injury to small pulmonary vessels by the stiff wires used by interventional cardiologists in the process of guiding their catheters and balloons. Flash pulmonary edema or frank hemorrhage can also result from the sudden relief of a gradient across a stenotic PA [3]. In certain instances, the area beyond the stenosis is immediately faced with a significant increase in systolic and diastolic pressures resulting in such injury [4]. These types of injuries have been described in the adult literature for patients with chronic thromboembolic pulmonary hypertension [5], rarely in the pediatric population, and even less so from the anesthetic perspective of managing ventilation, oxygenation and maintaining the airway.

Our long-standing institutional approach to this patient population has been catheter-based rehabilitation via balloon angioplasty with or without stent therapy performed via percutaneous or hybrid approach. As such, we have a large patient population who has presented to both the hybrid interventional catheterization suites and hybrid operating room for procedures planned to augment their PA flow. The primary aim of this retrospective review was to determine the incidence of pulmonary hemorrhage associated with these procedures and the feasibility of predicting this outcome. The secondary aim was to discuss our novel anesthetic approach to a select group of these patients, involving the use of bronchial blockers to preemptively prevent contamination of bilateral bronchi and occlusion of the endotracheal tube.

\section{Methods}

Prior to any interventional procedure in the cardiac operating room or the interventional catheterization suite, the involved personnel representing teams from nursing, technicians, operators, anesthesia and perfusion meet and discuss the case. This pre-procedure discussion has been termed a "huddle". The goal of a huddle, held immediately prior to bringing a patient into a room, is to outline any equipment needed, any anticipated challenges and develop a plan for the post-procedure disposition.

The general approach to treating pulmonary hemorrhage during rehabilitation includes the following options, based on the severity of the bleeding: (1) increase the positive end-expiratory pressure on the ventilator, (2) suction the endotracheal tube, (3) consider termination of the procedure and reversal of heparin with protamine, (4) placement of epinephrine into the endotracheal tube to provide vascular constriction, (5) support via extracorporeal membrane oxygenation.
Given the problems encountered with pulmonary hemorrhage during pulmonary artery rehabilitation, it has become our practice to discuss the utilization of bronchial blockers in huddles. Our blocker of choice is the Arndt Endo-Bronchial Blocker ${ }^{\circledR}$, and depending on the size of the patient and the diameter of the endotracheal tube, this device may be placed either inside or alongside the endotracheal tube. These blockers are available in a variety of sizes including a $5 \mathrm{~F} 50 \mathrm{~cm}, 7 \mathrm{~F} 65 \mathrm{~cm}$ and $9 \mathrm{~F} 78 \mathrm{~cm}$; please see the included picture of this device. When placed inside the endotracheal tube the ability to pass suctioning catheters may be impeded, and in very small endotracheal tubes such placement may impede ventilation. The theoretical disadvantage of placing these devices alongside the endotracheal tube is associated trauma to the vocal cords caused by a rigid device over the course of a potentially lengthy case. Regardless of blocker placement either inside or outside the endotracheal tube, the bronchial positioning of these devices in the catheterization suite with the assistance of fluoroscopy is easily accomplished. In cases that have a high likelihood of hemorrhage, the placement of these devices is collaboratively discussed in the huddle, and placement of the blocker is planned from the outset.

Figure 1 depicts a bronchial blocker placed alongside the endotracheal tube and positioned in the right main-stem bronchus. In this position, the blocker may be left deflated throughout the procedure and inflated only if evidence of pulmonary hemorrhage develops. Figure 2 demonstrates a three-dimensional reconstruction of the right pulmonary artery with a bronchial blocker again positioned alongside the endotracheal tube.

After approval by our institutional review board, we reviewed the records of all patients who underwent PA rehabilitation between October 1, 2010 and October 1, 2015.

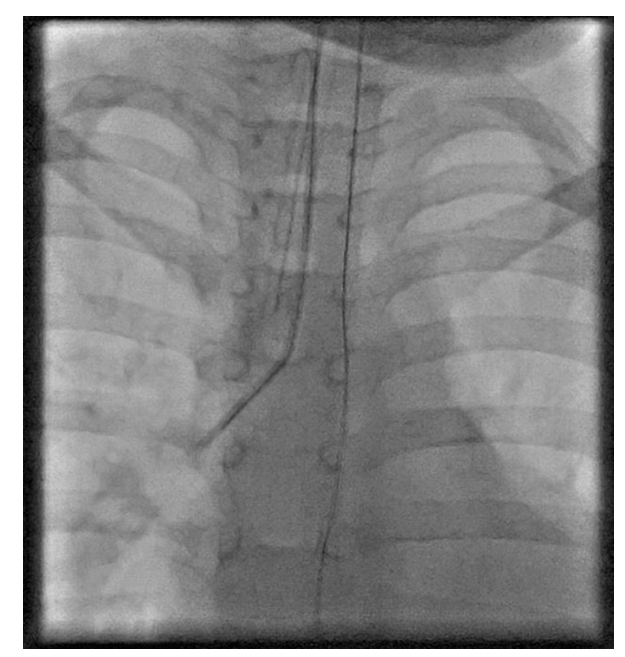

Fig. 1 Bronchial blocker placed in right main-stem bronchus 


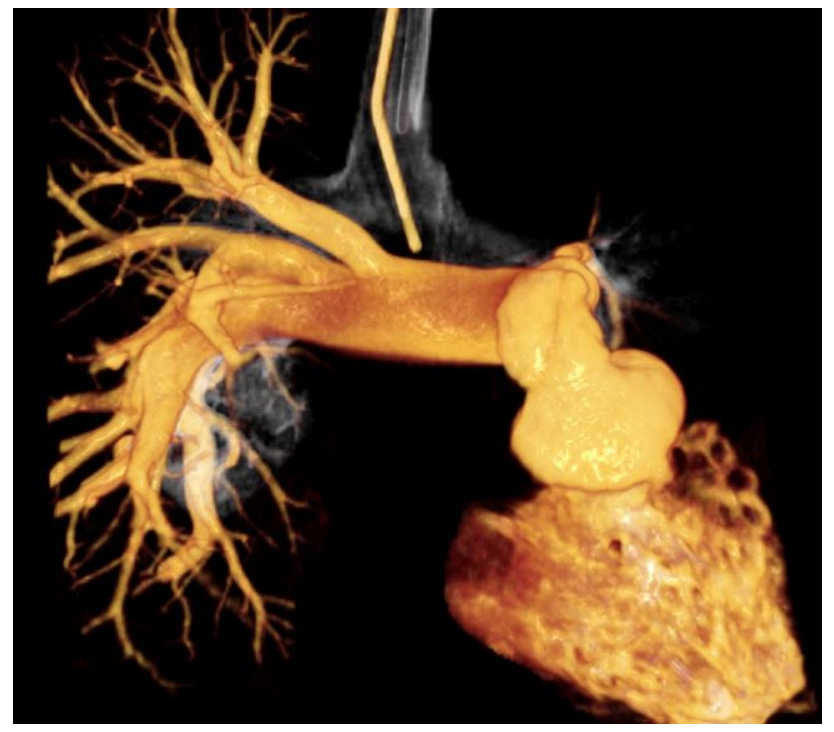

Fig. 2 Three-dimensional reconstruction of bronchial blocker placed in left main-stem bronchus

Patients with single ventricle physiology were excluded. Data collected included age, gender, diagnosis, procedure, anesthetic techniques, use of bronchial blockers, number of PA branches dilated, incidence and number of stents used, length of the procedures, post procedure intubation versus extubation, length of ventilator support postoperatively (if remained intubated) and incidence of cardiac intensive care (CTICU) unit admission post procedure. In addition, we collected hemodynamic data during the procedures, including opening and ending right ventricle (RV) to femoral artery (FA) pressure gradient, opening fraction inspired oxygen $\left(\mathrm{FiO}_{2}\right)$, opening and ending partial oxygen pressure $\left(\mathrm{PaO}_{2}\right)$, opening and ending $\mathrm{PaO}_{2}$ to $\mathrm{FiO}_{2}$ ratio and opening and ending alveolar to arterial oxygen content ratio $\left(\mathrm{AaDO}_{2}\right)$. Bleeding and pulmonary hemorrhage complications were detected either intra-procedurally as frank bleeding from the endotracheal tube or post procedure by chest X-ray.

Patient, procedure, and outcome characteristics were summarized using means with standard deviations for continuous data, and counts with percentages for categorical data. Change in pressure ratios from before to after the intervention was assessed using paired $t$ tests. Patients with complete data on study covariates were included in a multivariable model predicting bleeding complications. The model included pressure ratios obtained prior to the intervention, due to significant missing data on the change in these ratios from before to after the intervention. The feasibility of predicting bleeding complications according to patient and procedure characteristics was evaluated using receiver operating characteristics (ROC) analysis, with the area under the ROC curve (AUC) summarizing the predictive value of the multivariable logistic model. $p<0.05$ was considered statistically significant, and analyses were performed using Stata/IC 13.1 (College Station, TX: StataCorp, LP).

\section{Results}

After excluding all single ventricle patients, a total of 105 patients (age $7 \pm 8$ years, range 3 months to 41 years) were included in the analysis, of whom 76 had a diagnosis of TOF and 29 had other preoperative diagnoses. Other diagnoses included TGA $(n=10)$, truncus arteriosus $(n=5)$, isolated PA stenosis $(n=11)$, and DORV with TGA $(n=3)$. Other characteristics of the sample are summarized in Table 1. In total, 28 (27\%) procedures had bleeding complications, of which 15 had bleeding through the endotracheal tube. Bronchial blockers were used in 11 cases, including 7 of the 28 cases with complications. There were 13 patients who remained intubated after the procedure, including 9 of the cases with complications. Patients were transferred to the ICU in 23 cases, including 16 of the cases with complications.

The majority of patients had TOF. In its severest forms, the ventricular septal defect cannot be closed or only partially closed (fenestrated VSD closure). In bivariate analysis, the proportion of cases with pulmonary hemorrhage was higher among patients with open or fenestrated VSDs Table 2 (15 of 39; 38\%) than among patients with complete VSD closure $(13$ of $66 ; 20 \% ; p=0.036$; odds ratio of 2.5 ; 95\% CI 1.1-6.2).

The RV to FA ratio, a measure of systemic to RV pressure, was $0.9 \pm 0.3$ prior to intervention $(n=102)$, and $0.7 \pm 0.2$ after intervention $(n=82)$. Among the 82 patients with data on RV to FA ratio before and after intervention, the average change in this ratio was $-0.2 \pm 0.2(p<0.001)$. The ratio of $\mathrm{PaO}_{2}$ to $\mathrm{FiO}_{2}$ was $333 \pm 95$ prior to intervention $(n=95)$, and $262 \pm 167$ after intervention $(n=66)$. Among 61 patients with data collected before and after intervention, the average change in this ratio was $-62 \pm 171(p=0.006)$. The ratio of $\mathrm{SpO}_{2}$ to $\mathrm{FiO}_{2}$ was $419 \pm 69$ prior to intervention $(n=92)$, and $226 \pm 141$ after intervention $(n=66)$. Among 58 patients with data collected before and after intervention, the average change in this ratio was $-186 \pm 146(p<0.001)$.

In multivariable logistic regression $(n=85$ cases with complete data; Table 3 ), only the number of branches dilated was independently associated with the odds of complications at the stated significance threshold (OR 1.7; 95\% CI $1.1-2.5 ; p=0.020$ ), although the overall predictive utility of the model was fair (ROC AUC $=0.77$ ).

Neither the specific type of intervention (i.e. standard balloon angioplasty, cutting balloon angioplasty or stent implantation) nor location of the intervention (i.e. hilar distal vasculature versus more central pulmonary artery) 
Table 1 Characteristics of study sample $(N=105)$

\begin{tabular}{|c|c|c|c|}
\hline Variable & $\begin{array}{l}\text { Cases with complete } \\
\text { data }(n)\end{array}$ & Mean (SD) & $N(\%)$ \\
\hline Age (y) & 105 & $7(8)$ & \\
\hline Weight (kg) & 105 & $26(25)$ & \\
\hline TOF diagnosis & 105 & & $76(72 \%)$ \\
\hline Open or fenestrated VSD & 105 & & $39(37 \%)$ \\
\hline Branches dilated & 105 & $3(2)$ & \\
\hline Stent placed & 105 & & $60(57 \%)$ \\
\hline Case time $(\mathrm{m})$ & 99 & $248(85)$ & \\
\hline Bronchial blocker used & 105 & & $11(10 \%)$ \\
\hline \multicolumn{4}{|l|}{$\mathrm{PaO}_{2} / \mathrm{FiO}_{2}$ ratio } \\
\hline Pre-intervention & 95 & $333(95)$ & \\
\hline Post-intervention & 66 & $262(167)$ & \\
\hline Change & 61 & $-62(171)$ & \\
\hline \multicolumn{4}{|l|}{$\mathrm{SpO}_{2} / \mathrm{FiO}_{2}$ ratio } \\
\hline Pre-intervention & 92 & $419(69)$ & \\
\hline Post-intervention & 66 & $226(141)$ & \\
\hline Change & 58 & $-186(146)$ & \\
\hline \multicolumn{4}{|l|}{$\mathrm{RV} / \mathrm{FA}$ ratio } \\
\hline Pre-intervention & 102 & $0.9(0.3)$ & \\
\hline Post-intervention & 82 & $0.7(0.2)$ & \\
\hline Change & 82 & $-0.2(0.2)$ & \\
\hline Complications noted & 105 & & $28(27 \%)$ \\
\hline Bleeding from endotracheal tube & 105 & & $15(14 \%)$ \\
\hline Remained intubated postoperatively & 105 & & $13(12 \%)$ \\
\hline Transferred to ICU & 105 & & $23(22 \%)$ \\
\hline
\end{tabular}

Table 2 Open or fenestrated VSD and pulmonary hemorrhage

\begin{tabular}{lccr}
\hline & Hemorrhage & $\begin{array}{l}\text { No hemor- } \\
\text { rhage }\end{array}$ & Total \\
\hline VSD open & 13 & 19 & 32 \\
VSD fenestrated & 2 & 5 & 7 \\
VSD closed & 13 & 53 & 66 \\
Total & 28 & 77 & 105 \\
\hline
\end{tabular}

Table 3 Multivariable logistic regression of complications during PA rehabilitation procedures of study sample $(N=85)$

\begin{tabular}{llll}
\hline Variable & OR & $95 \% \mathrm{CI}$ & $p$ \\
\hline Age $(\mathrm{y})$ & 1.1 & $0.9-1.4$ & 0.301 \\
Weight $(\mathrm{kg})$ & 1.0 & $0.9-1.0$ & 0.633 \\
TOF diagnosis & 0.6 & $0.1-2.6$ & 0.478 \\
Open or fenestrated VSD & 1.2 & $0.3-4.6$ & 0.795 \\
Branches dilated & 1.7 & $1.1-2.5$ & 0.020 \\
Stent placed & 0.5 & $0.2-1.6$ & 0.274 \\
Case time (m) & 1.0 & $1.0-1.0$ & 0.829 \\
$\mathrm{PaO}_{2} / \mathrm{FiO}_{2}$ ratio pre-intervention & 1.0 & $1.0-1.0$ & 0.432 \\
$\mathrm{SpO}_{2} / \mathrm{FiO}_{2}$ ratio pre-intervention & 1.0 & $1.0-1.0$ & 0.992 \\
$\mathrm{RV}_{\mathrm{FA} \mathrm{ratio}}$ pre-intervention & 7.7 & $0.5-120.7$ & 0.147 \\
\hline
\end{tabular}

was predictive or independently associated with a bleeding complication.

\section{Discussion}

As the surgical treatments for patients with complex congenital heart disease have improved, more patients are returning to the interventional cardiac catheterization suite in need of increased pulmonary artery flow. These patients are often clinically challenging and typically return for multiple procedures in attempt to augment their pulmonary blood flow. These catheter based treatments have an associated high degree of morbidity and mortality [4]. From our results, $27 \%$ of these patients had bleeding complications (including $14 \%$ who had bleeding from the endotracheal tube), and $22 \%$ were admitted to the CTICU. This is consistent with the published literature citing a high degree of morbidity for these types of procedures [6].

In an attempt to improve outcomes in the event of a catastrophic pulmonary hemorrhage, we have selectively opted to place bronchial blockers at the start of these cases. The utilization of bronchial blockers for the purpose of containing pulmonary hemorrhage in the setting of planned 
pulmonary artery rehabilitation is not described in the literature. In our cohort of 105 patients, 11 had a blocker preemptively placed at the beginning of the case. Figures 1 and 2 respectively demonstrate an angiogram and a three-dimensional reconstruction of a blocker placed into the right mainstem bronchi while the right pulmonary artery was undergoing rehabilitation. Given the presence of fluoroscopy, it is straightforward to reposition a blocker into either the right or left main stem bronchus as the procedure progresses.

An alternative to placing a blocker might involve purposefully intubating the right or left main stem bronchus while rehabilitating the opposite side. This approach using a cuffed endotracheal tube may provide protection from contamination of the ventilated lung in the event of a massive hemorrhage. It does, however, have several drawbacks including the likely isolation of the upper right lobe in the event of a right main stem intubation. Also, it can be challenging to position the endotracheal tube in the left main stem during an emergency. The approach of preemptively positioning an endotracheal tube in the right or left main stem bronchus might significantly reduce ventilation in patients that otherwise have no reperfusion injury. The advantage of the bronchial blocker is that it can be quickly deployed as needed and when deflated it has no negative impact on ventilation.

It is beyond the scope of this review to conclude that bronchial blockers should be placed in any given patient prior to pulmonary artery rehabilitation. Rather, we simply wish to advance the possible benefits of this technique for patients at risk of pulmonary hemorrhage. Preventing contamination of the bronchi on the side opposite of the rehabilitation may allow for continued ventilation and oxygenation in the setting of a catastrophic hemorrhage.

Of course, no technique is without inherent risks. The contrary argument to placing such blocking devices details potential trauma to the vocal cords, potentially caused by rigid blockers sitting alongside endotracheal tubes in small patients over the course of a lengthy rehabilitation. Furthermore, if the blocker succeeds in preventing hemorrhage into the contralateral lung, it is possible that clot formation in the affected bronchus could solidify and result in loss of patency of that bronchus.

Our bivariate analysis support that the presence of an open VSD or intended fenestrated VSD patch increases the risk for pulmonary hemorrhage compared to those with complete VSD closure. This makes clinical sense as those patients with open or fenestrated VSDs tend to be those with the most severe forms of CHD. Specifically, their pulmonary vasculature is so underdeveloped or hypoplastic that complete VSD closure is not feasible. It is these same patients that typically require the most aggressive interventions during pulmonary rehabilitation. This physiology, combined with an acute successful procedural outcome, can result in an immediate increase in segmental pulmonary blood flow often with immediate increase in distal pulmonary pressure. This combination explains why this subset of patients is at increased risk for pulmonary hemorrhage and our data can help in appropriate pre-procedural planning and patient/family counseling.

In addition, our results indicate that dilating multiple branches has a significant predictive value of complications, $(p=0.02)$. The mean number of branches dilated was 3 with a standard deviation of 2 . In a bivariate analysis, the optimal threshold for the number of branches dilated predicting occurrence of complications was 2 . This calculation is of limited utility as it is approximately equivalent to saying that dilating multiple branches is riskier than dilating just one branch, which is a conclusion already supported by the multivariable model results. There is no further evidence from these data as to how many multiple branches dilated (if $\geq 2$ ) would pose the highest risk. It would seem logical though to suggest that the more branches one dilates, the more the risk of a bleeding complication. This would in part be due to the need for moving a relatively stiff interventional wire from one segment to the next, each time allowing for the rare but real possibility of a distal wire injury to the pulmonary vasculature. A larger sample size is needed to reach any such predictive conclusion. Counter intuitively; case time did not predict complications $(p=0.829)$. Finally, the RV/FA ratio pre-intervention trended toward but did not reach statistical significance $(p=0.147)$.

The practice of pulmonary artery rehabilitation has expanded considerably with the technical advances in catheterization equipment and the collective experience of pediatric interventional cardiologists. The availability of stents, covered stents, high-pressure balloons and cutting balloons has increased the number of therapeutic options. Alongside more invasive and interventional approaches to what was once considered a static and untreatable disease, the risk of significant hemorrhage is a real and potentially catastrophic side effect. This review details our experience with these cases and offers a novel airway management approach in attempt to minimize the perils associated with a massive pulmonary hemorrhage.

\section{Compliance with Ethical Standards}

Conflict of interest There was no external funding used in the completion of this project. None of the authors listed on this manuscript have any financial conflicts of interest.

Ethical Approval All procedures were performed in accordance with the ethical standards of Nationwide Children's Hospital which are entirely consistent with the 1964 Helsinki declaration and its later amendments and other comparable ethical standards.

Informed Consent The need for informed consent was waived by the Nationwide Children's Hospital institutional review board because this study was a retrospective review and all patients were de-identified. 


\section{References}

1. Soquet $\mathrm{J}$ et al (2017) Achievements and limitations of a strategy of rehabilitation of native pulmonary vessels in pulmonary atresia, ventricular septal defect, and major aortopulmonary collateral arteries. Ann Thorac Surg 103(5):1519-1526

2. Chen Q et al (2016) Multistage pulmonary artery rehabilitation in patients with pulmonary atresia, ventricular septal defect and hypoplastic pulmonary artery. Eur J Cardiothorac Surg 50(1):160-166

3. Inami $\mathrm{T}$ et al (2015) Incidence, avoidance, and management of pulmonary artery injuries in percutaneous transluminal pulmonary angioplasty. Int J Cardiol 201:35-37
4. Arnold LW et al (1988) Transient unilateral pulmonary edema after successful balloon dilation of peripheral pulmonary artery stenosis. Am J Cardiol 62(4):327-330

5. Andreassen AK et al (2013) Balloon pulmonary angioplasty in patients with inoperable chronic thromboembolic pulmonary hypertension. Heart 99(19):1415-1420

6. Yacouby S, Meador M, Mossad E (2014) Lung reperfusion injury in patients after balloon angioplasty for pulmonary artery stenosis. J Cardiothorac Vasc Anesth 28(3):502-505 